\title{
Desempenho de Métodos de Preenchimento de Falhas em Dados de Evapotranspiração de Referência para Região Oeste do Paraná
}

\author{
Tharsos Hister Giovanella $^{1}$ (D), Fabrício Correia de Oliveira ${ }^{1}$ (D), Vitor Alex de Alves Marchi ${ }^{2}$, \\ Júlia Tluszcz ${ }^{3}$ \\ ${ }^{1}$ Curso de Agronomia, Universidade Tecnológica Federal do Paraná, Santa Helena, PR, Brasil. \\ ${ }^{2}$ Curso de Ciências da Computação, Universidade Tecnológica Federal do Paraná, Santa \\ Helena, PR, Brasil. \\ ${ }^{3}$ Curso de Engenharia Elétrica, Universidade Tecnológica Federal do Paraná, Medianeira, PR, \\ Brasil.
}

Recebido em: 16 de Janeiro de 2021 - Aceito em: 30 de Abril de 2021

\begin{abstract}
Resumo
A ocorrência de falhas em leituras de variáveis meteorológicas em estações de superfície pode comprometer a consistência das séries históricas, inviabilizando ou prejudicando sua utilização. Neste sentido, o objetivo deste trabalho foi avaliar o desempenho de métodos para preenchimento de falhas em séries históricas de dados de evapotranspiração de referência (ETo), considerando a região oeste do Paraná. Foram utilizadas duas estações como referência, estação teste 1 (Foz do Iguaçu) e estação teste 2 (Marechal Cândido Rondon). Foram avaliadas cinco alternativas para preenchimento de falhas: Ponderação Regional com quatro estações vizinhas (PR-4), Ponderação Regional com uma estação vizinha (PR-1), Inverso do Quadrado da Distância (IQD), Mínimos Quadrados (MQ) e dados provenientes do NASA/POWER (NP). Os métodos foram comparados estatisticamente por meio da correlação de Pearson $(r)$, concordância de Willmott (d), coeficiente de eficiência Nash-Sutcliffe (CE), Raiz Quadrada do Erro Médio (RMSE) e Erro Médio Absoluto (EMA). Nas condições em que foram realizadas a pesquisa, todos os métodos avaliados apresentaram desempenho adequado, indicando potencial para serem utilizados como método de preenchimento de falhas. O método IQD se destacou entre os demais, pois suas estimativas foram classificadas como "correlação forte" ( $r=0,868$ - estação teste 1 e $r=0,887$ - estação teste 2) e "adequados" (CE = 0,999 - em ambas estações testes). Além de valores de "d" muito próximos de 1 e baixos erros de estimativas $\left(\operatorname{RMSE}=1,22 \mathrm{~mm} \mathrm{~d}^{-1}\right.$ e EMA $=0,82 \mathrm{~mm} \mathrm{~d}^{-1}$, estação teste 1 ; $\operatorname{RMSE}=0,87 \mathrm{~mm} \mathrm{~d}^{-1}$ e EMA $=0,68 \mathrm{~mm} \mathrm{~d}^{-1}$, estação teste 2 ), sendo o método recomendado para preenchimento de falhas em séries históricas de dados de ETo.
\end{abstract}

Palavras-Chave: interpolação espacial, NASA/POWER, ponderação regional, recursos hídricos.

\section{Performance of Filling Gaps in Reference Evapotranspiration Data for the Western Region of Paraná}

\begin{abstract}
The occurrence of failures in the readings of meteorological variables at surface stations can compromise the consistency of the historical series, making their use unfeasible or impairing. In this sense, the objective of this work was to evaluate the performance of methods for filling gaps in historical series of reference evapotranspiration data (ETo), considering the western region of Paraná. Two stations were used as reference, test station 1 (Foz do Iguaçu) and test station 2 (Marechal Cândido Rondon). Five alternatives for filling in gaps were evaluated: Regional Weighting with four neighboring stations (PR-4), Regional Weighting with one neighboring station (PR-1), Inverse Square Distance (IQD), Least Square (MQ) and data from NASA/POWER (NP). The methods were statistically compared using Pearson's correlation $(r)$, Willmott's agreement $(d)$, Nash-Sutcliffe efficiency coefficient (CE), Root Mean Square Error (RMSE) and
\end{abstract}

Autor de correspondência: Tharsos Hister Giovanella, tharsoshg@gmail.com. 
Mean Absolute Error (EMA). Under the conditions in which the research was carried out, all the methods evaluated showed adequate performance, indicating the potential to be used as a method of filling in gaps. The IQD method stood out among the others, since their estimates were classified as "strong correlation" ( $r=0.868$ - test station 1 and $r=0.887$ - test station 2) and "adequate" ( $\mathrm{CE}=0.999$ - in both test stations). In addition to "d" values very close to 1 and low estimation errors $\left(\right.$ RMSE $=1.22 \mathrm{~mm} \mathrm{~d}^{-1}$ and EMA $=0.82 \mathrm{~mm} \mathrm{~d}^{-1}$, test station 1 ; RMSE $=0.87 \mathrm{~mm} \mathrm{~d}^{-1}$ and EMA $=0.68 \mathrm{~mm} \mathrm{~d}^{-1}$ ), being the recommended method for filling series failures in historical ETo data series.

Keywords: spatial interpolation, NASA/POWER, regional weighting, water resources.

\section{Introdução}

A evapotranspiração é a perda de água na forma de vapor, da superfície evaporante para a atmosfera, ocorrendo por meio de dois processos: evaporação de superfícies inanimadas e transpiração de superfícies vivas (Reichardt e Timm, 2012). A evapotranspiração de referência (ETo) é aquela que ocorre em condições padronizadas, depende apenas de elementos meteorológicos, sendo também considerada um elemento meteorológico (Allen et al., 1998).

Estimativas acuradas de ETo são extremamente relevantes para gestão adequada dos recursos hídricos e planejamento das atividades agrícolas. Entre os exemplos de aplicação de ETo, destacam-se: dimensionamento e manejo de sistemas de irrigação, determinação da taxa de crescimento dos vegetais, realizar balanço hídrico em diferentes escalas de tempo e espaço, compreensão da relação solo-planta-atmosfera, análise de microclimas e interações com superfícies e estudos sobre drenagem do solo (Allen et al., 2005; Almorox et al., 2015; Santos et al., 2017).

A ETo pode ser determinada de maneira direta, por meio do uso dos lisímetros, que são equipamentos de custo e manutenção elevados, o que limita a utilização destes a instituições de pesquisa e universidades (Valipour, 2015), ou indireta, utilizando equações ou modelos empíricos, que necessitam de dados meteorológicos para sua aplicação (Almorox et al., 2015; Djaman et al., 2015). Entretanto, frequentemente ao analisar séries de dados meteorológicos, observam-se períodos com inconsistências ou falhas nas leituras (Bier e Ferraz, 2017).

A falta de continuidade nas séries de dados pode levar a conclusões errôneas em processos que dependem diretamente do clima, como a agricultura e planejamento de uso dos recursos naturais (Coutinho et al., 2018). Desta forma, tornam-se necessárias pesquisas que avaliem formas para se preencher os intervalos de dados faltantes das séries históricas, de modo a melhorar as estimativas ETo, e consequentemente os processos em que a variável é utilizada.

Devido a pertinência de se obter séries históricas de dados meteorológicos para o planejamento de atividades dependentes do clima, diversas pesquisas têm sido realizadas visando avaliar maneiras viáveis para o preenchimento de falhas (Brubacher et al., 2020). Os métodos mais utilizados podem ser divididos em diferentes abordagens: regressões lineares (simples ou múltiplas); modelos base- ados em aprendizagem de máquinas (redes neurais artificiais e máquina de vetores de suporte); e, interpoladores espaciais (ponderação regional, inverso do quadrado da distância, método comum no Reino Unido, vizinho natural, Spline e Krigagem) (Bier e Ferraz, 2017; Brubacher et al., 2020; Coutinho et al., 2018).

Mais recentemente, com a popularização de plataformas globais de dados meteorológicos como a ERAInterim do ECMWF e NASA/POWER, estes bancos de dados vêm sendo utilizado para preenchimento de séries históricas de estações meteorológicas de superfícies, apresentando resultados satisfatórios em diversas aplicações (Aparecido et al., 2019; Aparecido et al., 2020; Duarte e Sentelhas, 2020).

Apesar da importância da ETo como variável componente do balanço hídrico, a maioria das pesquisas que avaliam desempenho de métodos para preenchimento de falhas estão concentradas em dados de chuva (Brubacher et al., 2020; Srivastava et al., 2020). Neste sentido, o objetivo deste trabalho foi avaliar o desempenho de métodos para preenchimento de falhas em séries históricas de dados de evapotranspiração de referência, considerando a região oeste do Paraná.

\section{Material e Métodos}

A região de estudo é localizada no oeste do estado do Paraná. A região apresenta apenas duas estações meteorológicas automáticas da rede de estações do Instituto Nacional de Meteorologia (INMET), uma localizada em Foz do Iguaçu, PR (estação teste 1) e outra em Marechal Cândido Rondon, PR (estação teste 2). Estas foram selecionadas como estações testes, pois são as únicas estações meteorológicas localizadas na região oeste do Paraná. Além disso, apresentam diversas falhas em suas séries históricas.

Visando aplicar diferentes métodos de preenchimento de falhas, foi necessário utilizar dados de outras seis estações meteorológicas próximas à região oeste do Paraná, visto que, como mencionado anteriormente, só existem duas estações da rede INMET nesta região.

As estações testes de Foz do Iguaçu e Marechal Cândido Rondon foram utilizadas como referência para comparar o desempenho dos métodos de preenchimento de falhas, representadas no mapa como estação teste $1 \mathrm{e}$ estação teste 2, respectivamente. As demais estações fo- 
ram utilizadas como fonte de dados para aplicar os métodos de preenchimento de falhas (Fig. 1).

A localização e o código das estações testes utilizadas na pesquisa podem ser observadas na Tabela 1 .

Inicialmente, foram adquiridos e organizados dados de elementos meteorológicos necessários para estimativa de ETo. Para isso, foram utilizadas séries históricas de um período de cinco anos, iniciando em 01/01/2015 até 31/12/ 2019. Foram selecionados dados que coincidiam no tempo entre as diferentes estações meteorológicas utilizadas, correspondendo a aproximadamente $75 \%$ dos dados da série histórica. Os dados meteorológicos retirados de cada estação foram: temperatura máxima, temperatura mínima, radiação solar global, umidade relativa máxima, umidade relativa mínima e velocidade do vento. Os dados meteorológicos retirados da plataforma NASA/POWEER foram: temperatura máxima, temperatura mínima, radiação solar global, umidade relativa média e velocidade do vento. $\mathrm{O}$ saldo de radiação foi determinado a partir dos valores de radiação solar global e os dados de temperatura máxima e temperatura mínima foram utilizados para determinar a temperatura média (Allen et al., 1998).

Tabela 1 - Localização e o código das estações meteorológicas utilizadas na pesquisa.

\begin{tabular}{ccccccc}
\hline Estação meteorológica & Código INMET & Lat. $\left(^{\circ}\right)$ & Lon. $\left({ }^{\circ}\right)$ & Alt. $(\mathrm{m})$ & DH-ET1 (m) & DH-ET2 (m) \\
\hline Estação Teste 1 (Foz do Iguaçu-PR) & A846 & $-25,60$ & $-54,48$ & 235,09 & - & 122985,00 \\
Estação Teste 2 (M. C. Rondon-PR) & A820 & $-24,53$ & $-54,02$ & 392,07 & 122985,00 & - \\
Cidade Gaúcha-PR & A869 & $-23,36$ & $-52,93$ & 365,79 & 211657,00 & 174635,00 \\
Itaquiraí-MS & A752 & $-23,45$ & $-54,18$ & 337,82 & 232829,00 & 120486,00 \\
Maringá-PR & A835 & $-23,41$ & $-51,93$ & 548,51 & 353283,00 & 248024,00 \\
Nova Tebas-PR & A822 & $-24,44$ & $-51,96$ & 656,24 & 287792,00 & 211584,00 \\
Planalto-PR & A855 & $-25,72$ & $-53,75$ & 399,10 & 83363,00 & 131260,00 \\
Sete Quedas-MS & A751 & $-23,97$ & $-55,02$ & 398,40 & 181389,00 & 119141,00 \\
\hline
\end{tabular}

*Lat $\left({ }^{\circ}\right)$ - latitude; Lon. $\left({ }^{\circ}\right)$ - longitude; DH-ET1 - distância horizontal até a estações teste 1; DH-ET2 - distância horizontal até a estações teste 2.

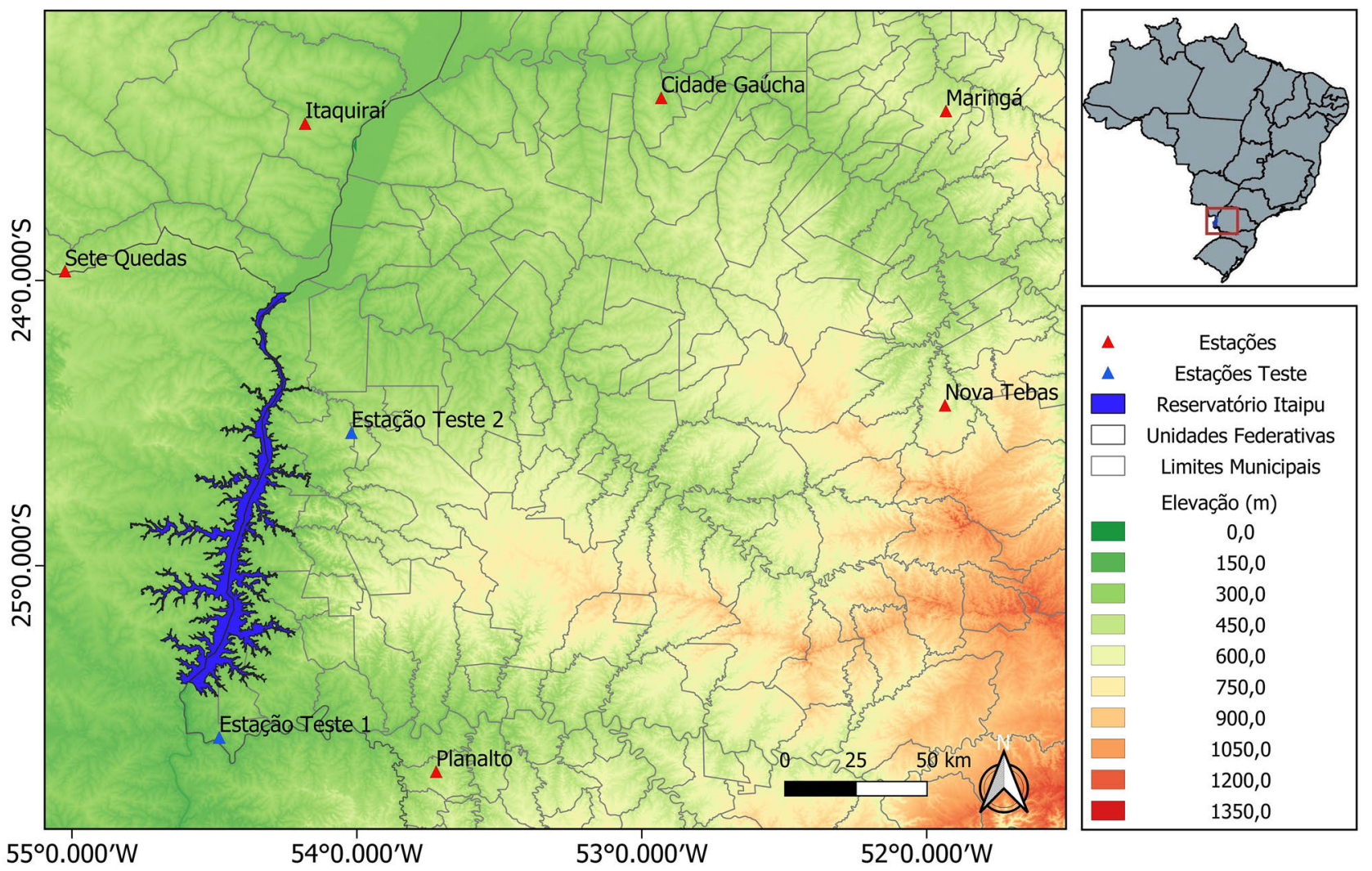

Figura 1 - Estações meteorológicas e área de estudo utilizada na pesquisa com representação espacial das elevações. 
A ETo diária foi determinada utilizando a equação de Penman-Monteith (Eq. (1)) (Allen et al., 1998).

$$
\operatorname{ETo}_{P M}=\frac{0,408 \cdot s \cdot(R n-G)+\gamma \cdot\left(\frac{900}{T+273}\right) \cdot U_{2} \cdot\left(e_{s}-e_{a}\right)}{s+\gamma \cdot\left(1+0,34 \cdot U_{2}\right)}
$$

em que $T$ - temperatura média $\left({ }^{\circ} \mathrm{C}\right) ; R n$ - saldo de radiação $\left(\mathrm{MJ} \mathrm{m}^{-2} \mathrm{~d}^{-1}\right) ; s$ - declividade da curva de pressão $\left(\mathrm{kPa}^{\circ}{ }_{-}\right.$ $\left.\mathrm{C}^{-1}\right) ; e_{s}$ - pressão de saturação de vapor $(\mathrm{kPa}) ; e_{a}$ - pressão de vapor $(\mathrm{kPa}) ; U_{2}$ - velocidade média do vento $\left(\mathrm{m} \mathrm{s}^{-1}\right)$; $\gamma$ - constante psicrométrica $\left(\mathrm{kPa}^{\circ} \mathrm{C}^{-1}\right)$; e, $G$ - fluxo de calor no solo ( $\mathrm{MJ} \mathrm{m}^{-2} \mathrm{~d}^{-1}$ ). Os valores de ETo estimados com os dados das estações testes corresponderam aos valores observados (EToOBS), considerados referência. Os valores ETo calculados nas demais estações foram utilizados como fonte de dados para os métodos de preenchimento de falhas.

Foram selecionadas cinco alternativas de preenchimento de falhas para serem avaliadas: Ponderação Regional com quatro estações vizinhas (PR-4) (Eq. (2)), Ponderação Regional com uma estação vizinha (PR-1) (Eq. (2)) (Bier e Ferraz, 2017; Diaz et al., 2018; Mello et al., 2017), Inverso do Quadrado da Distância (IQD) (Eq.(3)), Mínimos Quadrados (MQ) (Brubacher et al., 2020) e dados provenientes do NASA/POWER (NP) (Srivastava et al., 2020).

$$
\begin{gathered}
\operatorname{ETo}_{P R}=\frac{\sum_{i=1}^{n}\left(\frac{p_{\text {med }}}{p_{\text {med }-i}} \cdot X_{i}\right)}{N_{p}} \\
\operatorname{ETo}_{I Q D}=\frac{\sum_{i=1}^{n}\left(\frac{1}{d_{i}^{2}} \cdot X_{i}\right)}{\sum_{i=1}^{n}\left(\frac{1}{d_{i}{ }^{2}}\right)}
\end{gathered}
$$

em que $P R$ - índice que corresponde aos valores de ETo estimados pelo método de ponderação regional, podendo ser com quatro estações vizinhas (PR-4) ou uma estação vizinha (PR-1); $I Q D$ - índice que corresponde aos valores de ETo estimados pelo inverso do quadrado da distância; $p_{\text {med }}$ - média mensal de dados da estação teste; $p_{\text {med-i }}$ média do dado da estação vizinha de ordem $i ; X_{i}$ - dado ocorrido na estação vizinha de ordem $i$ no mês de ocorrência da falha na estação teste; $d_{i}{ }^{2}$ - distância Euclidiana entre a estação de ordem $i$ e a estação teste; e, $N_{p}$ - número de estações vizinhas utilizadas no cálculo, neste caso foram as quatro mais próximas para PR-4 e a mais próxima para PR-1.

O MQ foi utilizado para gerar um ajuste de curva para estimar ETo em cada estação teste a partir de dados das estações vizinhas. Para isso, foi utilizado o software $\mathrm{R}$ e dados de dois anos (2018 e 2019) dos cinco anos utiliza- dos na pesquisa. Para a estação teste 1 foram três estações vizinhas (Eq. (4)), enquanto que para a estação teste 2 foram quatro estações vizinhas (Eq. (5)). O número de estações vizinhas consideradas foi definido de acordo com o desempenho do ajuste de curva.

$$
\begin{gathered}
E T o_{E T 1}=1,916+0,10142 \cdot N T+0,20479 \cdot P \\
+0,29272 \cdot S Q \\
E T o_{E T 2}=1,46106+0,21257 \cdot I+0,14852 \cdot M \\
+0,22361 \cdot P+0,24881 \cdot S Q
\end{gathered}
$$

em que os índices ET1 e ET2 indicam ETo interpolada para estação teste 1 e estação teste 2; $N T$ - ETo observada na estação de Nova Tebas; $P$ - ETo observada na estação de Planalto; $S Q$ - ETo observada na estação de Sete Quedas; $I$ - ETo observada na estação de Itaquiraí; e, $M$ - ETo observada na estação de Maringá.

Os valores de ETo determinados com os dados provenientes do NASA/POWER foram comparados aos valores observados nas estações teste. O conjunto de dados utilizados nas estimativas dos métodos de preenchimento de falhas avaliados pode ser observado na Tabela 2.

A avaliação do desempenho dos métodos de preenchimento de falhas foi realizada tomando-se os valores de ETo das estações testes como referências. Foram utilizados os seguintes indicadores estatísticos: correlação de Pearson, $r$ (Eq. 6); concordância de Willmott, $d$ (Eq. (7)) (Willmott et al., 2011); coeficiente de eficiência Nash-Sutcliffe, $C E$ (Eq. (8)) (Van Liew et al., 2007); Raiz Quadrada do Erro Médio, RMSE (Eq. (9)); e, Erro Médio Absoluto,

Tabela 2 - Conjunto de dados utilizados nas estimativas dos métodos de preenchimento de falhas avaliados.

\begin{tabular}{cc}
\hline \multicolumn{2}{c}{ Estação Teste 1 (Foz do Iguaçu) } \\
$\begin{array}{c}\text { Métodos de preenchimento de } \\
\text { falhas }\end{array}$ & $\begin{array}{c}\text { Código do INMET das estações } \\
\text { vizinhas utilizadas }\end{array}$ \\
\hline PR-1 & A855 \\
PR-4 & A869, A820, A855, A751 \\
IQD & A820, A869, A752, A835, A822, A855 \\
e A751 \\
MQ & A822, A855 e A751 \\
\hline Estação Teste 2 (Marechal Cândido Rondon) \\
\hline PR-1 \\
PR-4 & A846 \\
IQD & A869, A820, A822 e A751 \\
MQ & e A746, A869, A752, A835, A822, A855 \\
& A752, A835, A855 e A751 \\
\hline
\end{tabular}

*PR-4 - Ponderação Regional com quatro estações vizinhas; PR-1 - Ponderação Regional com uma estação vizinha; IQD - Inverso do Quadrado da Distância; MQ - Ajuste de Mínimos Quadrados; e, NP - dados provenientes do NASA/POWER. 
EMA (Eq. (10)) (Fox, 1981).

$$
\begin{gathered}
r=\frac{\sum_{i=1}^{n}\left(X_{i}-\bar{X}\right)\left(Y_{i}-\bar{Y}\right)}{\sqrt{\left[\sum_{i=1}^{n}\left(X_{i}-\bar{X}\right)^{2}\right]\left[\sum_{i=1}^{n}\left(Y_{i}-\bar{Y}\right)^{2}\right]}} \\
d=1-\frac{\sum_{i=1}^{n}\left(Y_{i}-X_{i}\right)^{2}}{\sum_{n=1}^{n}\left[\left(\left|Y_{i}-\bar{X}\right|\right)+\left(\left|X_{i}-\bar{X}\right|\right)\right]^{2}} \\
C E=1-\left(\frac{\sum_{i=1}^{n}\left(Y_{i}-X_{i}\right)^{2}}{\sum_{i=1}^{n}\left(Y_{i}-\bar{Y}\right)^{2}}\right) \\
R M S E=\sqrt{\frac{\sum_{i=1}^{n}\left(Y_{i}-X_{i}\right)^{2}}{n}} \\
E M A=\frac{1}{n} \sum_{i=1}^{n}\left|Y_{i}-X_{i}\right|
\end{gathered}
$$

em que: $Y_{i}$ - valor observado na estação teste, $\mathrm{mm} \mathrm{d}^{-1} ; X_{i}$ valor estimado pelo método de preenchimento de falhas, $\mathrm{mm} \mathrm{d}^{-1} ;-Y$ - valor médio observado na estação teste, $\mathrm{mm} \mathrm{d}^{-1} ;-X$ - valor médio estimado pelo método de preenchimento de falhas; e, $n$ - número de observações.

A análise dos resultados dos valores de " $r$ " pode seguir a seguinte classificação: correlação muito forte $(r>0,9)$; correlação forte $(0,9>r>0,7)$; correlação moderada $(0,7>r>0,5)$; correlação fraca $(0,5>r>0,3)$; correlação desprezível $(0,3>r>0)$ (Cunha et al., 2013). $\mathrm{O}$ índice " $d$ " varia de zero a um. O valor um indica perfeita concordância e zero indica nenhuma concordância (Willmott et al., 2011).

$\mathrm{O}$ indicador $C E$ pode ser utilizado para avaliar o desempenho dos métodos de preenchimento de falhas, sendo: perfeito $(C E=1)$; adequado $(C E>0,75)$; satisfatório $(0,75>C E>0,36)$; e, não satisfatório $(C E<0,36)$. Esse indicador avalia o desvio dos valores observados em relação ao desvio dos valores estimados, quanto mais próximo de um, menor é o desvio dos valores estimados (Van Liew et al., 2007). O EMA indica a diferença média entre os valores medidos e os valores estimados, sendo valores mais próximos de zero considerados ideais. O RMSE funciona de maneira semelhante, sendo mais sensível a des- vios maiores. Portanto, quanto maior o RMSE, maiores serão os desvios dos valores simulados (Chai e Draxler, 2014).

\section{Resultados e Discussão}

Em relação aos valores médios de ETo, observa-se que na estação teste 1 (Foz do Iguaçu) a média \pm desvio padrão dos valores foi de $4,56 \pm 1,73 \mathrm{~mm} \mathrm{~d}^{-1}$. A média obtida pelos métodos PR-4, PR-1, IQD, MQ e NP foram $4,54 \pm 1,34 ; 4,56 \pm 1,65 ; 4,76 \pm 1,39 ; 4,70 \pm 0,81 ; \mathrm{e}, 4,04$ $\pm 1,76 \mathrm{~mm} \mathrm{~d}^{-1}$ respectivamente, representados pela linha cinza. A linha preta indica a mediana dos valores (Fig. 2).

A média \pm desvio padrão dos valores de ETo observados para estação teste 2 (Marechal Cândido Rondon) foi de $4,94 \pm 1,50 \mathrm{~mm} \mathrm{~d}^{-1}$. A média obtida pelos métodos PR-4, PR-1, IQD, MQ e NP foram 4,94 $\pm 1,50 ; 4,94 \pm$ 1,$35 ; 4,63 \pm 1,32 ; 5,39 \pm 1,13 ; \mathrm{e}, 4,63 \pm 1,52 \mathrm{~mm} \mathrm{~d}^{-1}$ respectivamente, representados pela linha cinza. A linha preta indica a mediana dos valores (Fig. 3).

Em relação à média \pm desvio padrão dos valores de ETo provenientes dos diferentes métodos de preenchimentos de falhas avaliados, observa-se que os valores médios foram muito semelhantes à referência (EToOBS) em ambas estações testes. Além disso, o desvio padrão da média também foi numericamente semelhante quando se compara o desvio padrão da média de ETo provenientes dos métodos de preenchimentos de falhas e observado nas estações testes. O método de MQ, nas duas estações testes, resultou maior valor médio e menor variabilidade dos valores de ETo, em relação aos demais métodos avaliados, indicando baixa variabilidade em suas estimativas.

Em relação aos valores dos indicadores estatísticos $(r, d, C E, R M S E$ e EMA) provenientes da comparação entres os valores de ETo estimadas e observados nas estações testes, observa-se que todos os métodos avaliados

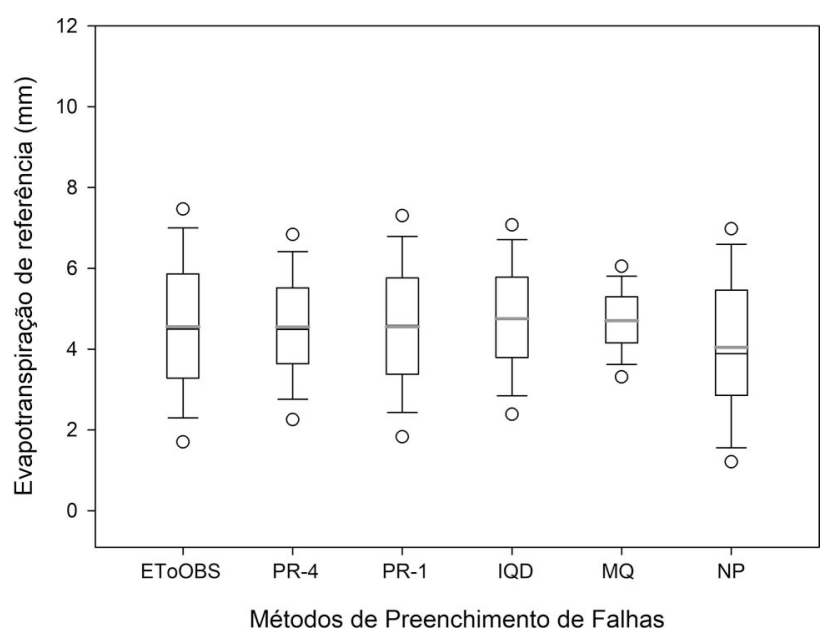

Figura 2 - Valores de ETo observados e estimados pelos cinco métodos de preenchimento de falhas (PR-4, PR-1, IQD, MQ e NP) na estação teste 1 (Foz do Iguaçu). 


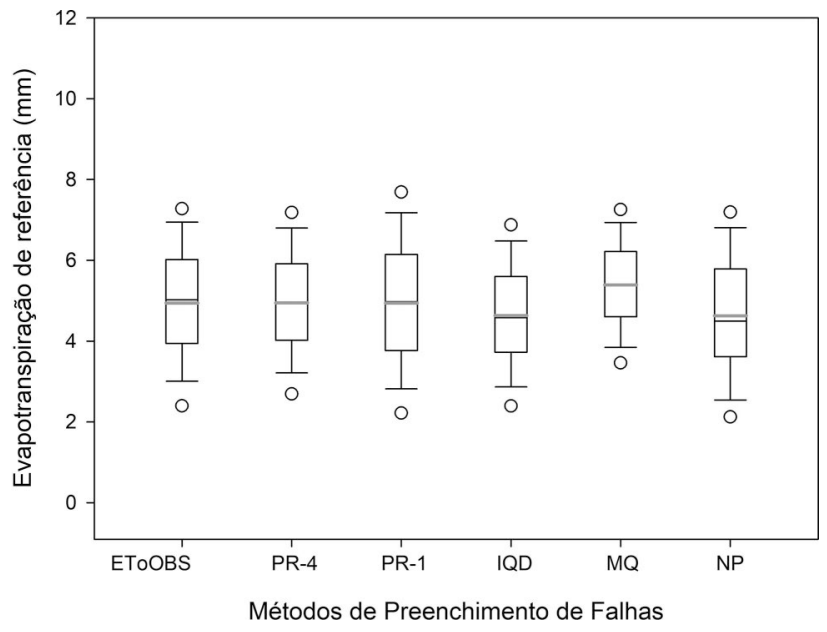

Figura 3 - Valores de ETo observados e estimados pelos cinco métodos de preenchimento de falhas (PR-4, PR-1, IQD, MQ e NP) na estação teste 2 (Marechal Cândido Rondon).

apresentaram desempenho adequado, indicando potencial para serem utilizados como método de preenchimento de falhas de dados de ETo (Tabela 3).

Considerando classificação da correlação de Pearson, os resultados das estimativas de ETo foram classificados como "correlação forte", de modo que apresentam valores de " $r$ " acima de 0,8 (Cunha et al., 2013). Os cinco métodos avaliados apresentaram concordância entre os valores de ETo estimados e observados em ambas estações testes, uma vez que os valores de " $d$ " foram próximos da unidade (Willmottet al., 2011).

Tabela 3 - Valores da correlação de Pearson $(r)$, concordância de Willmott $(d)$, coeficiente de eficiência Nash-Sutcliffe $(C E)$, Raiz Quadrada do Erro Médio (RMSE) e Erro Médio Absoluto (EMA) para estação teste 1 (Foz do Iguaçu) e estação teste 2 (Marechal Cândido Rondon).

$\begin{array}{llllll}\text { Métodos de preenchimento de } & r & d & C E & R M S E & E M A\end{array}$ falhas

\begin{tabular}{llllll}
\hline \multicolumn{7}{c}{ Estação teste 1 (Foz do Iguaçu) } \\
\hline PR-4 & 0,855 & 0,999 & 0,498 & 1,22 & 0,88 \\
PR-1 & 0,845 & 0,999 & 0,403 & 0,52 & 0,94 \\
IQD & 0,868 & 0,999 & 0,999 & 1,22 & 0,82 \\
MQ & 0,844 & 0,999 & 0,999 & 1,31 & 1,01 \\
NP & 0,886 & 0,9998 & 0,999 & 1,38 & 1,05 \\
\hline Estação teste 2 (Marechal Cândido Rondon) & & \\
\hline PR-4 & 0,869 & 0,999 & 0,645 & 0,89 & 0,68 \\
PR-1 & 0,808 & 0,999 & 0,386 & 0,37 & 0,90 \\
IQD & 0,887 & 0,999 & 0,999 & 0,87 & 0,68 \\
MQ & 0,884 & 0,999 & 0,999 & 0,95 & 0,67 \\
NP & 0,873 & 0,999 & 0,999 & 1,00 & 0,75 \\
\hline
\end{tabular}

*PR-4 - Ponderação Regional com quatro estações vizinhas; PR-1 - Ponderação Regional com uma estação vizinha; IQD - Inverso do Quadrado da Distância; MQ - Ajuste de Mínimos Quadrados e NP - dados provenientes do NASA/POWER
Considerando as duas estações testes, os métodos IQD, MQ e NP apresentaram valores muito próximos a um $(C E=0,999)$, sendo classificados como "adequados". Os métodos PR-4 e PR-1 foram classificados como "satisfatórios" (Van Liew et al., 2007). O menor RMSE foi observado pelo método PR-1, em ambas as estações testes. Na estação teste 1, o método IQD apresentou menor EMA $\left(0,82 \mathrm{~mm} \mathrm{~d}^{-1}\right)$. Enquanto que na estação teste 2, o menor valor foi observado com o método MQ $\left(0,67 \mathrm{~mm} \mathrm{~d}^{-1}\right)$. Embora os erros tenham sido relativamente baixos, os maiores erros de estimativas foram reportados pelo método NP.

Para ambas estações testes, observa-se nesta pesquisa que com o aumento do número de estações vizinhas utilizadas, a correlação entre os valores observados e os valores estimados aumentou, além disso, proporcionou redução do indicador EMA. O método PR-1 proporcionou baixos valores de RMSE, devido à proximidade entre a estação vizinha e a estação teste. No caso da PR-4, onde foram utilizadas 4 estações vizinhas, a correlação aumentou, porém o RMSE aumentou devido ao incremento de pontos amostrais. Comportamento semelhante aconteceu com o método $\mathrm{MQ}$, em que a utilização de mais pontos amostrais favoreceu para um melhor ajuste de curva, consequentemente, reduzindo os erros de estimativa. No entanto, o aumento dos pontos amostrais sem a devida regularidade de distribuição espacial causa redução da correlação entre dados estimados e observados (Chen, 2012).

Fazendo um balanço entre todos os indicadores estatísticos utilizados, observa-se que o IQD se destacou entre os demais, em ambas estações testes, pois as adequações de suas estimativas foram classificadas como "correlação forte" ( $r=0,868$ - estação teste 1 e $r=0,887$ - estação teste 2) e "adequados" ( $C E=0,999$ - em ambas estações testes). Os valores de " $d$ " obtidos foram de 0,999 , indicando concordância quase perfeita. Além dos baixos índices de erros de estimativas $\left(R M S E=1,22 \mathrm{~mm} \mathrm{~d}^{-1}\right.$ e $E M A=0,82 \mathrm{~mm}$ $\mathrm{d}^{-1}$, na estação teste 1 e $R M S E=0,87 \mathrm{~mm} \mathrm{~d}^{-1} \mathrm{e}$ $E M A=0,68 \mathrm{~mm} \mathrm{~d}^{-1}$, estação teste 2).

O desempenho do método IQD pode ser bastante influenciado pela uniformidade e abrangência da distribuição dos pontos amostrais (Brubacher et al., 2020; Murara, 2019). Possivelmente, os erros de estimativas (RMSE e $E A M)$ não foram menores devido à baixa distribuição $\mathrm{e}$ uniformidade espacial das estações vizinhas, utilizadas como pesos no cálculo das estimativas de ETo (Murara, 2019).

O método IQD baseia-se na atribuição de pesos às variáveis de acordo com a proximidade entre os pontos amostrais. Pontos próximos tendem a ser mais semelhantes em relação aos pontos mais afastados. Os pesos atribuídos determinam a influência do valor de cada ponto amostral no valor interpolado. Portanto, para obter desempenhos satisfatórios nas estimativas, é necessário que os 
pontos amostrais sejam próximos e uniformemente distribuídos (Chen, 2012).

A distribuição irregular ocasiona maiores erros de estimativa, principalmente com o aumento da distância entre os pontos amostrais (Brubacher et al., 2020). Além disso, o método IQD considera somente a distância entre pontos, negligenciando outros fatores responsáveis pela possível variação espacial dos valores de ETo, como a variações de altitude dos pontos amostrais (Murara, 2019). Assim, o principal fator limitante para encontrar métodos de preenchimento de falhas que apresentam elevado desempenho para a região oeste do Paraná é a distribuição extremamente não uniforme das estações meteorológicas, fator fundamental para obter elevado desempenho de estimativa (Bier e Ferraz, 2017; Brubacher et al., 2020). Uma vez que as estações testes 1 e 2 estão localizadas na fronteira entre o Brasil e o Paraguai, desta forma, as estações vizinhas estão localizadas apenas do lado brasileiro, reduzindo a uniformidade de distribuição dos pontos amostrais e a representatividade dos valores interpolados, consequentemente, redução do desempenho dos métodos.

Assim, o método IQD que utilizou 7 estações vizinhas proporcionou melhores valores de correlação com erros mais baixos devido ao grande número de pontos amostrais e melhor distribuição desses pontos. Deste modo, o aumento dos pontos amostrais proporcionou maior representatividade espacial dos valores de ETo, reduzindo os erros de estimativa decorrentes das variações de altitude. Resultados semelhantes foram obtidos por Hodam et al. (2017) comparando o desempenho do método IQD com o método de Krigagem para estimativas de ETo na Índia. Os autores observaram que o IQD apresentou melhor desempenho, sendo recomendado para interpolação espacial de ETo em escala mensal. Saraiva et al. (2017) avaliando o desempenho de IQD, Krigagem e Spline tensionado para estimativas de ETo, no estado do Espírito Santo, Brasil, observaram que o IQD apresentou melhor desempenho para estimativas diárias de ETo.

Os dados provenientes da base de dados NASA/ POWER, por serem derivados de sensoriamento remoto, possuem menor resolução espacial em relação aos dados oriundos de estações meteorológicas automáticas de superfície. Entretanto, os resultados apresentaram boa correlação com os dados observados nas estações testes de superfície, indicando potencial para calibração das variáveis, podendo reduzir os erros de estimativa (RMSE e $E A M)$. Considerando a distribuição global da base de dados, a facilidade de obtenção dos dados e os últimos avanços visando incrementar a acurácia destas fontes de dados abertos, o preenchimento de falhas em séries de dados meteorológicos utilizando o método NP se torna uma opção tecnicamente viável. Embora necessite calibração para consolidação do método como preenchimento de falhas de variáveis meteorológicas, diversas pesquisas utilizando essa base de dados vem sendo realizadas
(Aboelkhai et al., 2019; Duarte e Sentelhas, 2020; Monteiro et al., 2017; Srivastava et al., 2020).

\section{Conclusões}

Considerando a análise gráfica dos valores de ETo e indicadores estatísticos utilizados, observou-se que todos os métodos avaliados apresentaram desempenho adequado, indicando potencial para serem utilizados como método de preenchimento de falhas de dados de ETo. O IQD se destacou entre os demais, em ambas estações testes, pois suas estimativas apresentaram correlações fortes, ajustes adequados e baixos erros, sendo o método recomendado para preenchimento de falhas em séries históricas de dados de $\mathrm{ETo}$, considerando a região oeste do Paraná.

\section{Agradecimentos}

Agradecemos à Universidade Tecnológica Federal do Paraná e a Fundação Araucária pelo apoio financeiro.

\section{Referências}

ABOELKHAIR, H.; MORSY, M.; EL AFANDI, G. Assessment of agroclimatology NASA POWER reanalysis datasets for temperature types and relative humidity at 2 meter against ground observations over Egypt. Advances in Space Research, v. 64, n. 1, p. 129-142, 2019.

ALLEN, R.G.; PEREIRA, L.S.; RAES, D.; SMITH, M. Crop evapotranspiration: Guidelines for computing crop water requirements. Rome: FAO, 1998.

ALLEN, R.G.; WALTER, I.A.; ELLIOTT, R.L.; HOWELL, T.A.; ITENFISU, D.; JENSEN, M.E.; SNYDER, R.L. The ASCE standardized reference evapotranspiration equation. Reston: ASACE, 2005.

ALMOROX, J.; QUEJ, V.H.; MARTÍ, P. Global performance ranking of temperature-based approaches for evapotranspiration estimation considering Köppen climate classes. Journal of Hydrology, v. 528, n. 9, p. 514-522, 2015.

APARECIDO, L.E.O.; ROLIM, G.S.; DE MORAES, J.R.S.C.; TORSONI, G.B.; DE MENESES, K.C.; COSTA, C.T.S. Acurácia da reanálise ERA-Interim do ECMWF e sua aplicação na estimativa da deficiência hídrica no estado do Paraná, Brasil. Revista Brasileira de Meteorologia, v. 34, n. 4, p. 515-528, 2019.

APARECIDO, L.E.O.; ROLIM, G.S.; MORAES, J.R.S.C. Validation of ECMWF climatic data, 1979-2017, and implications for modelling water balance for tropical climates. International Journal of Climatology, v. 40, n. 15, p. 120, 2020.

BIER, A.A.; FERRAZ, S.E.T. Comparação de metodologias de preenchimento de falhas em dados meteorológicos para estações no Sul do Brasil. Revista Brasileira de Meteorologia, v. 32, n. 2, p. 215-226, 2017.

BRUBACHER, J.P.; OLIVEIRA, G.G.; GUASSELLI, L.A. Preenchimento de falhas e espacialização de dados pluviomé- 
tricos: desafios e perspectivas. Revista Brasileira de Meteorologia, v. 35, n. 4, p. 615-629, 2020.

CHAI, T.; DRAXLER, R.R. Root mean square error (RMSE) or mean absolute error (MAE)? - Arguments against avoiding RMSE in the literature. Geoscientific Model Development, v. 7, n. 3, p. 1247-1250, 2014.

CHEN, F.W; LIU, C.W. Estimation of the spatial rainfall distribution using inverse distance weighting (IDW) in the middle of Taiwan. Paddy and Water Enviroment, v. 10, n. 3, p. 209-222, 2012.

COUTINHO, E.R.; DA SILVA, R.M.; MADEIRA, J.G.F.; COUTINHO, P.R. de O. dos S.; BOLOY, R.A.M.; DELGADO, A.R.S. Application of artificial neural networks (ANNs) in the gap filling of meteorological time series. Revista Brasileira de Meteorologia, v. 33, n. 2, p. 317-328, 2018.

CUNHA, F.F.; MAGALHÃES, F.F.; CASTRO, M.A. Método para estimativa da evapotranspiração de referência para Chapadão do Sul - MS. Engenharia na Agricultura, v. 21, n. 2, p. 159-172, 2013

DIAZ, C.C.F.; PEREIRA, J.A.S.; NÓBREGA, R.S. Comparação de dados estimados por dois diferentes métodos para preenchimento de falhas de precipitação pluvial na bacia do rio Pajeú, Pernambuco, Brasil. Revista Brasileira de Climatologia, v. 22, p. 324-339, 2018.

DJAMAN, K.; BALDE, A.B.; SOW, A.; MULLER, B.; IRMAK, S.; N'DIAYE, M.K.; MANNEH, B.; MOUKOUMBI, Y.D.; FUTAKUCHI, K.; SAITO, K. Evaluation of sixteen reference evapotranspiration methods under sahelian conditions in the Senegal River Valley. Journal of Hydrology: Regional Studies, v. 3, p. 139-159, 2015.

DUARTE, Y.C.N.; SENTELHAS, P.C. NASA/POWER and DailyGridded weather datasets - How good they are for estimating maize yields in Brazil? International Journal of Biometeorology, v. 64, n. 3, p. 319-329, 2020.

FOX, D.G. Judging air quality model performance. Bulletin of the American Meteorological Society, v. 62, n. 2, p. 599$609,1981$.

HODAM, S.; SARKAR, S.; MARAK, A.G.R.; BANDYOPADHYAY, A.; BHADRA, A. Spatial interpolation of reference evapotranspiration in India: comparison of IDW and Kriging Methods. Journal of The Institution of Engineers (India): Series A, v. 98, n. 4, p. 511-524, 2017.

MELLO, Y.R.; KOHLS, W.; OLIVEIRA, T.M.N. Uso de diferentes métodos para o preenchimento de falhas em estações pluviométricas. Boletim de Geografia, v. 35, n. 1, p. 112$121,2017$.

MONTEIRO, L.A.; SENTELHAS, P. C.; PEDRA, G. U. Assessment of NASA/POWER satellite-based weather system for
Brazilian conditions and its impact on sugarcane yield simulation. International Journal of Climatology, v. 3, n. 38 , p. $1560-1570,2017$.

MURARA, P. Técnica de interpolação para a pesquisa em climatologia regional e agroclimatologia. Revista Brasileira de Climatologia, Edição Especial - Simpósio Brasileiro de Climatologia Geográfica - 2018, p. 106-126, 2019.

REICHARDT, K.; TIMM, L.C. Solo, Planta e Atmosfera. 2. ed. São Paulo: Manole, 2012.

SANTOS, L.C.; JOSÉ, J.V; ALVES, D.S.; NITSCHE, P.R.; REIS, E.F.; BENDER, F.D. Space-time variability of evapotranspiration and precipitation in the State of Paraná, Brazil. Revista Ambiente e Água, v. 12, n. 5, p. 743-759, 2017.

SARAIVA, G.S.; BONOMO, R.; SOUZA, J.M. Avaliação de interpoladores geoestatísticos e determinísticos da evapotranspiração de referência diária para o estado do Espírito Santo. Revista Agro@Mbiente on-Line, v. 11, n. 1, p. 2130, 2017.

SRIVASTAVA, P.K.; SINGH, P.; MALL, R.K.; PRADHAN, R.K.; BRAY, M.; GUPTA, A. Performance assessment of evapotranspiration estimated from different data sources over agricultural landscape in Northern India. Theoretical and Applied Climatology, v. 140, n. 1-2, p. 145-156, 2020.

VALIPOUR, M. Temperature analysis of reference evapotranspiration models. Meteorological Applications, v. 22, n. 3, p. 385-394, 2015.

VAN LIEW, M.W.; VEITH, T.L.; BOSCH, D.D.; ARNOLD, J.G. Suitability of SWAT for the conservation effects assessment project: comparison on USDA agricultural research service watersheds. Journal of Hydrologic Engineering, v. 12, n. 2, p. 173-189, 2007.

WILLMOTT, C.J.; ROBESON, S.M.; MATSUURA, K. A refined index of model performance. International Journal of Climatology, v. 32, n. 13, p. 2088-2094, 2011.

\section{Endereços de Internet}

Endereços de internet NASA/POWER. Portal NASA Prediction Of Worldwide Energy Resources. Disponível em https:// power.larc.nasa.gov/. Acesso em: 26 jun. 2021.

License information: This is an open-access article distributed under the terms of the Creative Commons Attribution License (type CC-BY), which permits unrestricted use, distribution and reproduction in any medium, provided the original article is properly cited. 